\title{
Environnement \& Hydroélectricité : HydroES 2016 Synthèse de la conférence internationale organisée par la SHF les 16 et 17 mars 2016 à Grenoble
}

\author{
Agnès BARILLIER ${ }^{1}$, Guy CAIGNAERT ${ }^{2}$, Véronique GOURAUD ${ }^{3}$, Maria-Helena RAMOS ${ }^{4}$, Didier ROULT ${ }^{5}$, \\ Pierre-Louis VIOLLET ${ }^{6}$
}

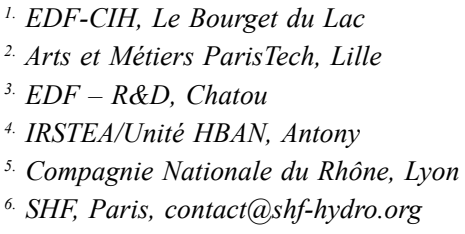

RESUME. - L'hydroélectricité est une source importante d'énergie renouvelable pour la production électrique en Europe et dans le monde. Tout en étant flexible et permettant le stockage de l'énergie, l'hydroélectricité facilite également l'intégration des énergies intermittentes au sein du système électrique. Le développement, l'opération et la manutention de la production hydroélectrique sont étroitement liés à des préoccupations croissantes concernant la durabilité environnementale, l'atténuation du changement climatique et la réponse aux besoins de la société en matière d'énergie. Des installations hydrauliques fiables et efficaces peuvent améliorer la performance hydroélectrique et l'acceptabilité environnementale des nouveaux projets, quand elles sont également prévues pour protéger l'environnement dans lequel elles opèrent. Ce document présente une synthèse de la conférence internationale HydroES 2016 - Hydroélectricité et environnement, organisée par la SHF et accueillie par INP ENSE3 à Grenoble, les 16-17 mars 2016. Il montre que la communauté scientifique et l'industrie hydroélectrique sont engagées dans l'évaluation des impacts environnementaux et sociaux de l'hydroélectricité et proactifs vers un avenir durable.

Mots-clés : Hydroélectrictié, impact environnemental

\section{Hydropower and Environmental Sustainability - HydroES 2016 Report on the Conference held by SHF in Grenoble on March 16-17, 2016}

\begin{abstract}
Hydropower is an important source of renewable energy for electrical production in Europe and throughout the world. Being flexible and allowing energy storage, it also facilitates the development of intermittent energy in electricity systems. The development, operation and maintenance of hydropower production are closely related to growing concerns on environment sustainability, climate change mitigation and meeting societal needs for energy. Reliable and efficient hydropower facilities can improve hydropower performance and the environmental acceptability of new projects when they are also planned to protect the environment in which they operate. This document presents a synthesis report of the international conference HydroES 2016 - Hydropower \& Environmental Sustainability, organized by SHF and hosted by INP ENSE3, in Grenoble, on 16-17 March 2016. It shows that the scientific community and the hydropower industry are committed to assessing environment and social impacts of hydropower and are proactive towards a sustainable future.
\end{abstract}

Key-words: Hydroelectricity, environmental impact

\section{INTRODUCTION}

15 ans après la mise en place de la Directive Cadre européenne sur l'Eau, ce colloque avait pour objectif de faire un point sur la prise en compte des aspects environnementaux et sociétaux dans les projets hydroélectriques. Sachant que des éléments de contexte nouveaux ont pris de l'ampleur :

— une meilleure connaissance des effets des changements climatiques sur les questions hydriques,

- la mise en oeuvre d'une politique de transition énergétique dans la plupart des pays européens, suite au développement des énergies renouvelables et de démarches de transition vers une économie bas-carbone et durable.
Il s'agit donc de lever la contradiction entre l'objectif de développer les énergies renouvelables et le souci de préserver les milieux aquatiques.

Ce colloque faisait suite à d'autres colloques organisés par la SHF sur l'hydroélectricité :

- Environnement et Hydroélectricté (colloque national, en français, 2010),

- Les stations de pompage hydrauliques (colloque international, 2012),

- La rénovation des installations hydroélectriques (colloque international, 2014). 
Le présent colloque s'est tenu dans les locaux de l'Ecole ENSE3 à Grenoble. Trois thèmes l'ont structuré :

- l'intégration socio-environnementale des installations hydroélectriques,

- les continuités écologiques et sédimentaires,

- les innovations technologiques en vue de réduire les impacts.

Le plan de la présente synthèse suit ces trois thèmes.

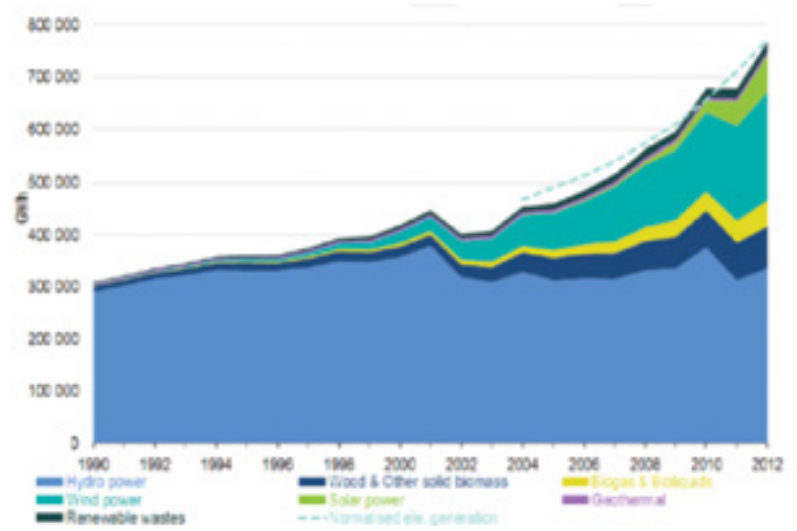

Figure 1 : Le développement des énergies renouvelables en Europe (extrait de la conférence introductive de P. Sagnes).

\section{L'INTÉGRATION SOCIO-ENVIRON- NEMENTALE DES INSTALLATIONS HYDROÉLECTRIQUES}

\section{L'hydroélectricité contribue au développement des énergies renouvelables}

L'hydroélectricité, par son caractère flexible et sa capacité de stockage d'eau, et donc d'énergie, est un soutien primordial au développement et au déploiement des énergies solaire et éolienne (figure 1). La mer du Nord, par exemple, représente pour l'Europe un gisement éolien considérable, et 95 GW de capacité de production éolienne offshore devraient y être installés d'ici 2030. Une capacité de stockage d'énergie de plusieurs dizaines de TWh serait ainsi nécessaire en Europe aux environs de 2050 (Harby \& al.). Certains pays, comme la Norvège qui dispose de centaines de grands réservoirs, s'estiment en capacité de contribuer de façon très significative à ce stockage.

Ce même contexte pousse aussi vers une plus grande flexibilité de la production hydroélectrique. Cette flexibilité se traduit en pratique par des éclusées ${ }^{1}$ (dont les conséquences sur l'environnement sont discutées dans la section 2 , et par un fonctionnement en mode transitoire et à charge partielle des machines (voir la section 3).

Le développement de l'hydroélectricité contribue par ailleurs en lui même aux objectifs de développement des énergies renouvelables. L'intégration de l'hydroélectricité au fil de l'eau modifie la part optimale entre énergies solaire et éolienne dans le mix énergétique et permet d'augmenter le taux de pénétration globale des énergies renouvelables (François et al.). Dans l'intégration énergie, eau, société et environnement, la question se pose toujours : faut-il privilégier les grands aménagements ou les petites chutes ? La réponse fait débat avec des impacts cumulés des petits

1. Fortes variations temporelles de débit liées au turbinage ouvrages qui peuvent s'avérer plus importants que ceux des grands aménagements (G. Petts). La réponse est sans doute dépendante des caractéristiques géographiques locales et des politiques régionales. Ainsi, en Ecosse, le développement de la petite hydroélectricité a été encouragé grâce à des tarifs de rachat attractifs (Wilmott, \& al.), moyennant des travaux permettant les continuités écologiques. Dans une région très spécifique comme la Guyanne, les pentes faibles des cours d'eau font qu'un grand aménagement va ennoyer des centaines de $\mathrm{km}^{2}$ de forêt et milieux humides, ce qui conduit plutôt à recommander le développement de chutes plus petites, susceptibles de noyer une moindre surface de forêt. Dans un pays comme la Norvège, la planification, la construction et la gestion des ouvrages hydroélectriques est encadrée par une démarche intégrant les aspects techniques, économiques, environnementaux et socio-politiques.

\section{L'eau, une ressource à partager entre les différents usages}

L'exemple de la Durance, rivière du sud-est de la France, est significatif d'un bassin soumis à de nombreuses contraintes économiques (produire de l'énergie autant que possible, tout en utilisant l'eau aussi pour l'agriculture et le tourisme), et environnementales (maintenir la capacité de pêcher, maintenir des débits écologiques et soutenir les étiages). Une communauté de gestion du bassin de la Durance a été constituée : les acteurs locaux se réunissent régulièrement, sur une longue période de temps, partageant une évaluation des rapports de force et motivées par l'obtention d'un compromis, sous la présidence d'un médiateur (Santoni).

Dans un autre exemple, celui du bassin du fleuve Sénégal, le rôle du Gestionnaire de l'Eau comme arbitre entre les différents usages (eau potable, énergie, agriculture, navigation, etc.) a été également souligné (Trouvat).

\section{Des systèmes de management environnemental pour les} projets hydroélectriques

Trouver localement l'équilibre entre les diverses contraintes auxquelles sont soumises les installations hydroélectriques peut être facilité par la mise en œuvre de systèmes de management environnemental, tel celui mis en place sur le Rhône (Coulon), qui repose sur la notion de risque environnemental, ou telle que l'application du protocole IHA qui permet d'évaluer la durabilité d'un projet hydroélectrique (Hydropower Sustainability Assessment Protocol). Ce protocole a été appliqué notamment au projet Romanche-Gavet en France (Branche), où il a été démontré que les impacts environnementaux et sociétaux peuvent être limités grâce à un engagement précoce et continu des parties prenantes.

\section{LES CONTINUITÉS ÉCOLOGIQUE ET SÉDIMENTAIRE}

La continuité sédimentaire, une composante fondamentale à intégrer et à restaurer

La modification du transit sédimentaire par les ouvrages a, dans le passé, trop souvent été vue comme une problématique locale : remplissage des retenues par les sédiments et diminution de la réserve utile, érosion du lit en aval.

De manière générale, la gestion sédimentaire est un des aspects de la problématique très complexe de la continuité écologique et doit être reconnue comme une composante fondamentale de la préservation des écosystèmes. 
Un tronçon de rivière fait partie d'un système longitudinal, et à ce titre il doit être replacé dans le contexte global de son bassin versant, mais les interactions avec les zones latérales, la présence de champs d'inondation, et les échanges avec les nappes souterraines jouent aussi un rôle prépondérant.

Ainsi, sur le Rhône, les aménagements réalisés à différentes époques ont considérablement réduit l'espace de mobilité latérale de la rivière. Des actions sur les marges alluviales visent à réactiver la dynamique fluviale. Les anciens épis Girardon, construits au XIXème siècle pour concentrer l'écoulement au milieu du lit, sont progressivement supprimés, et d'anciennes lônes (bras du Rhône) sont ré-ouvertes (S Reynaud).

Il ne suffit pas non plus de reproduire un système morphologiquement proche du système initial. A l'état naturel, les bancs de graviers évoluent et se renouvellent. Une stabilisation des formes morphologiques provoque un vieillissement du fond par dépôt de matières organiques, perte de perméabilité, croissance d'algues, et, par conséquent, conduit à une réduction de la biodiversité ( $\mathrm{G}$ Petts). La restauration de la dynamique fluviale et son évolution à long terme font partie des axes actuels de recherche qui mobilisent la communauté scientifique.

\section{La continuité écologique conditionnée par un continuum hydrologique, sédimentaire et migratoire}

Des avancées significatives ont été produites ces dernières décennies sur la réponse des espèces aux modifications de débit et à l'entrave de leurs conditions de circulation. La réponse aux dynamiques sédimentaires et morphologiques a été, en revanche, très peu étudiée. Les mortalités des macro-invertébrés liées aux taux élevés de matières en suspension - MES - (Bruno), le comportement in situ des poissons face au relargage de MES (Cattaneo) ou la réponse des communautés biologiques aux recharges sédimentaires (C. Steantzel) commencent à faire l'objet d'analyses approfondies.

En réponse au besoin d'une plus grande flexibilité de la production hydroélectrique ( $\mathrm{cf} \S 1$ ), de nombreuses études sont en cours sur l'impact écologique des éclusées et les mesures d'atténuation de ces impacts. Des outils permettent de quantifier les conditions d'habitat des espèces aquatiques pendant les éclusées (Le Coarer, Schneider) en fonction de différents modes de gestion. Les analyses du lien entre les caractéristiques des éclusées (débit maximal, gradient de débit...) et la végétation (Bejarono), les macro-invertébrés et les poissons, (Schmutz, Gouraud) permettent d'identifier les caractéristiques pénalisantes et d'évaluer l'efficacité de nouveaux modes de gestion des éclusées sur les communautés aquatiques. Les résultats montrent notamment une sensibilité plus importante des jeunes stades de développement. En raison de cette plus grande sensibilité, des études se concentrent, par exemple, sur la survie des œufs de saumon en lien avec différents scénarios de gestion (et leur traduction en coûts économiques) (Casas-Mulet) ou les risques d'échouage des jeunes individus (Schneider).

La conception des dispositifs de franchissement des barrages par les poissons, développés ces dernières décennies, est bien maitrisée pour la montaison des espèces (Vaxelaire), et de façon moindre pour la dévalaison. L'utilisation de ces dispositifs par les poissons peut être caractérisée à l'aide de capture au sein des dispositifs et de marquage (Benitez). Ces études permettent de déterminer les rythmes de migration selon les espèces et les saisons afin d'identifier leurs besoins migratoires. Si ces besoins sont évidents pour les espèces amphibiotiques $^{2}$, ils le sont moins pour les espèces holobiotiques $^{3}$, d'où la nécessité de caractériser en milieu naturel les capacités de dispersion d'espèces telles que la truite (Poulet) inféodée au voisinage de la majorité des ouvrages hydroélectriques.

\section{LES INNOVATIONS TECHNOLOGIQUES EN VUE DE RÉDUIRE LES IMPACTS}

La session 3 du colloque HydroES 2016 avait comme cadre les innovations technologiques associées à la limitation des impacts environnementaux et sociétaux des installations hydro-électriques.

Du côté " machines » de production d'hydro-électricité, les grandes tendances sont principalement associées aux conséquences de la variabilité de production dans le contexte du développement de l'utilisation des énergies éoliennes et solaires. Cela se traduit au niveau hydro-électrique par la nécessaire maîtrise des fonctionnements à vitesse variable et/ ou charges partielles, ainsi que par l'intégration de moyens de production plus distribués, en particulier dans le domaine des très faibles chutes.

Les défis identifiés concernent notamment la réhabilitation d'ouvrages anciens, ainsi que des avancées nécessaires dans les domaines :

- de la modélisation numérique des écoulements internes aux machines,

- de la réalisation d'expérimentations de validation de plus en plus fines,

- de l'instrumentation et du contrôle

- des matériaux, avec également la prise en compte nécessaire des interactions entre fluide et structures.

L'intérêt de la suppression des huiles et graisses minérales dans les organes de guidage et d'étanchéité des turbines, et la possible application de ce principe à la fois dans les nouveaux projets et dans les projets de réhabilitation de machines ont été mis en évidence.

L'existence d'une offre dans le domaine des machines axiales, adaptées aux très faibles chutes (VLH), et la compatibilité de ces machines avec le passage de poissons dévalants dans de très bonnes conditions, encore en cours d'amélioration grâce à des innovations technologiques, ont été démontrées.

Diverses présentations d'études de dispositifs et dispositions, destinés à limiter les impacts environnementaux des installations hydro-électriques, en particulier sur les poissons, ont été faites. Quelles que soient les échelles retenues (de l'échelle d'un pays à l'échelle d'un laboratoire), les présentations du colloque ont toutes démontré la nécessité de mener des expérimentations de qualité. Les principales avancées à retenir sont les suivantes :

- la mise en place d'outils et de lignes directrices pour limiter les impacts environnementaux des installations hydro-électriques sur les cours d'eau, et

- le développement de diverses technologies permettant de limiter les effets néfastes des installations hydro-électriques sur les poissons : doubles vis d'Archimède, effets

2. Un organisme est dit amphibiotique lorsque son cycle de vie se déroule dans deux milieux différents, en partie en eau marine et en partie en eau douce.

3. La migration holobiotique est particulière aux poissons migrateurs passant toute leur vie dans le même milieu (poissons dulçaquicoles ou poissons marins) et dans lequel ils effectuent leurs migrations 
- de macro-rugosités dans des passes à poissons à fentes verticales, utilisation d'exutoires de dévalaison associés à des grilles fines horizontales ou inclinées.

\section{REMERCIEMENTS}

Les auteurs remercient l'ensemble des membres du Comité de pilotage du colloque HyroES 2016 : Agnès Barillier (EDF), Jean-François Brun (SCP), Guy Caignaert, (ENSAM Lille) Anna Dupont (SHF), Claude Guilbaud (ARTELIA), Véronique Gouraud (EDF), Olivier Métais
(ENSE3-Grenoble), Jean-Luc Pigeon (Tractebel Ingéniérie), Maria-Helena Ramos (IRSTEA), Neda Sheibani (SHF), Olivier Teller (GE-ALSTOM), Pierre-Louis Viollet (SHF), ainsi que tous les participants du colloque.

\section{RÉFÉRENCES}

SHF (2016) - Hydropower and Environmental Sustainability HydroES 2016, Proceedings of the conference, Grenoble, 16-17 mars 2016. 217 ession du Comité Scientifique et Technique de la Société Hydrotechnique de France. 